\title{
Cloned Genes of Storage Proteins
}

Keith O. Elliston, Shahid Imran, and Joachim Messing

Waksman Institute (K.O.E, J.M.) and Center for Advanced

Biotechnology and Medicine (S.I.), Rutgers University,

Piscataway, NJ 08855-0759 USA

The compilation of the cloned and sequenced plant storage protein genes was performed on Release 14 of the EMBL database (Hamm \& Cameron, 1986). This list includes only those genes that have been cloned, sequenced, and submitted to the database.

We used the sequence analysis package from the Genetics Computer Group, GCG version 5.1 of Devereux et al. (1984) run on a Digital Systems Vax 11/785 minicomputer. Specifically, the program "Strings" was used to locate all sequences that had the character string storage or seed in their documentation. All entries that matched the string seed but not storage were checked to see if they were indeed storage protein genes; those that were not were eliminated. The data from this list of sequences was extracted and concatenated into a single file using the program "Fetch" of the GCG package. The relevant data of interest was then extracted using the program "Features" (written by S.I.), and formatted into a table using the program "make_table" (written by S.I. and K.O.E.). In order to eliminate duplications, the data from Genbank and EMBL databases were cross referenced using the spreadsheet program Excel on a MacIntosh SE. Additional searches were done using names of known storage proteins as strings; e.g., zein.

This approach can be generalized to formulate lists of any class of genes represented in the EMBL database. We should note that the EMBL database is directly accessible "online" via the BitNet computer network.

Acknowledgements: The computer analysis was supported by a Grant from the Department of Energy FCO2-84ER13210 to JM.

\section{References}

Hamm, G.H. \& G. N. Cameron. 1986. The EMBL data library. Nuc. Acids Res. 14:5-9. Devereux, J.B., P. Haeberli \& O. Smithies. A comprehensive set of sequence analysis program for the VAX. 1984. Nuc. Acids Res. 12: 387-395. 